\title{
A Statistical Metrology Approach to Compare the Quality of Optical Pulse Wave Signals
}

\author{
Janos Palhalmi ${ }^{1,2}$, Jan-Hein Broeders ${ }^{3}$ \\ ${ }^{1}$ EastWestData Ltd, Budapest, Hungary \\ ${ }^{2}$ DataSenseLabs Ltd, Budapest, Hungary \\ ${ }^{3}$ Analog Devices Inc, Eindhoven, The Netherlands
}

\begin{abstract}
Biometric metrology is becoming increasingly important as the wearable application specific biosensors are becoming capable of generating accurate raw signals representing certain vital states.

A comparative Biosignal Metrology method has been worked out (patent pending ID: P1900302) to answer questions arising from a biosensor and biosignal testing measurement science perspective.

In this specific work two high quality optical solutions (Analog Devices' : ADPD107 (EVAL-HCRWATCH) and ADPD188GGZ) were compared to analyze the deep data level similarities or differences between the photoplethysmography (PPG) raw biomedical signals generated by the two individual systems.

Based on the results we can conclude that the quality of the PPG signal recorded by the new ADPD188GGZ integrated optical module reaches the same performance level as the ADPD107 discrete module according to the newly established Biosignal Metrology Standards.
\end{abstract}

\section{Introduction}

With an increased focus on Home Health, Remote Monitoring and Prevention, a new market has been evolving around smart devices for tracking several vital parameters.

Besides the well-established ISO and IEC guidelines of the test criteria of the hardware components and circuitry boards, there is no standard protocol to generalize the comparative analysis of the raw biosignals recorded under different experimental or clinical circumstances.

In most of the cases when optical pulse volume signals, for example photoplethysmography (PPG) signals or sensor systems are compared, the heart rate properties are compared and analyzed [1], [2] instead of the raw or filtered datasets.

Clinical evaluation of pathological event recognition capability of wearable devices is based on high-level standards of study design and medical application [3], on the other hand the results of clinical tests cannot be used at the level of industrial decision making regarding the prototype or market ready device development.

In other words, there is a wide gap between the engineering level IEC and ISO testing protocols and the clinical evaluation of wearable cardiovascular solutions which can be filled by the validation of the raw biosignals compared to a reference or "gold standard" like it has been customary in other fields of metrology.

Therefore a Biosignal Metrology method has been developed (patent pending ID: P1900302) to facilitate the standardization of the validation of cardiovascular remote monitoring wearable solutions.

In this specific study the comparative analysis of a newly developed integrated optical module (Analog Devices Inc. ADPD188GGZ) was compared to an already proven solution as a "gold standard" of optical PPG sensors (Analog Devices Inc. ADPD107 analog optical front-end, designed for discrete optical systems).

\section{Methods}

\subsection{Test setup and data collection}

For the optical comparison, raw PPG readings were recorded, simultaneously with the ADPD188GGZ and ADPD107, over a period of 2 minutes. For the ADPD188GGZ setup, the standard evaluation board was used, where the ADPD107 was part of the optical system inside the Wearable demo platform (EVALHCRWATCH). Both systems have been controlled by Analog Devices' user-interface called "Application WaveTool". For the test, configuration settings were optimized to achieve the highest signal quality. We kept the configuration of the AFE's including the LED pulses, Timing and Transimpedance Gain within a specific range to achieve the same power consumption on both systems to get a fair comparison (see Table 1.). 
Table 1.

\begin{tabular}{lll}
\hline Text & ADPD188GGZ & ADPD107 \\
\hline $\begin{array}{l}\text { Power } \\
\text { consumption } \\
(\mathrm{mW})\end{array}$ & 5.124 & 5.188 \\
$\begin{array}{l}\text { Sampling } \\
\text { frequency }(\mathrm{Hz})\end{array}$ & $100-500$ & $100-500$ \\
LEDcurrent $(\mathrm{mA})$ & 130.02 & 64.89 \\
AFEWidth $(\mu \mathrm{s})$ & 3 & 3 \\
PulseWidth $(\mu \mathrm{s})$ & 2 & 2 \\
Pulse Offset $(\mu \mathrm{s})$ & 32 & 25 \\
AFE Offset $(\mu \mathrm{s})$ & 23 & 16 \\
AFE Fine Offset & 125 & 250 \\
$(\mathrm{~ns})$ & & \\
\hline
\end{tabular}

The table shows an ADPD188GGZ LED current, which is twice as high, versus the LED current in the ADPD107 setup. The reason is, that the Photo Diode Surface of the Integrated solution is smaller compared to the surface of the discrete solution, so we had to compensate for this. With 2 LEDs, driven from a $3 \mathrm{~V}$ supply this adds $156 \mu \mathrm{W}$ to the overall power dissipation what is almost negligible compared to the total power dissipation. We sampled the ADC at a rate of $100 \mathrm{~Hz}$, what is common in a wearable system. In addition, we measured also at $500 \mathrm{~Hz}$ sampling rate, what is often used for systems with clinical performance.

The data recording was taken under the same circumstances as a normal smart watch or fitness tracker, having the optical sensor attached to the top of the wrist. Since the microcirculation and vasoconstriction properties can be slightly different between the subdermal layers of the dominant hand and the non-dominant hand, the recordings were repeated on both wrists by both optical systems. Datasets collected from the left and right wrist were analyzed and compared carefully, to avoid position specific influence on the signal quality. PPG datasets were recorded on 11 different users (subjects), while seated and, under the same ambient light intensity conditions.

\section{Results}

To support a peak detection algorithm, baseline fluctuations within the PPG raw data can easily be subtracted and filtered. In parallel high signal quality around the peaks are needed at the raw data level to extract the targeted features. That is the reason why in this study we focused on the comparative analysis of the major frequency bands around the peaks of the PPG signals measured by the "gold standard" ADPD107 and the new integrated ADPD188GGZ optical module. Major components of the signals were not modified, except the very slow baseline fluctuations $(<0.25 \mathrm{~Hz})$ and the high frequency components $(>40 \mathrm{~Hz})$ which were filtered.

Wavelet coherence and correlation-based comparisons were computed to compare similarity of the two signals within the most dominant frequency ranges.

Figure 1 shows almost identical patterns of the results of the two PPG systems at the level of individual waveforms and their averages.
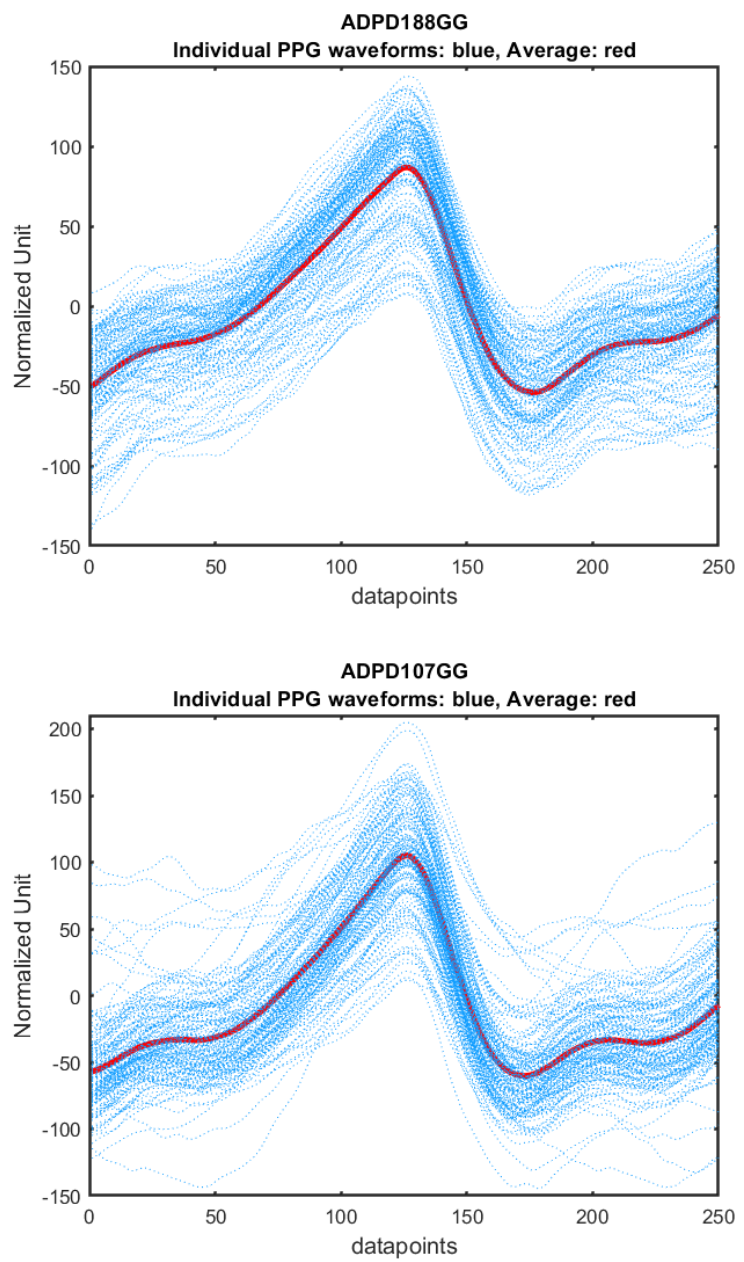

Figure 1. Individual PPG waveforms $( \pm 125$ datapoints around the local maximum) were extracted and plotted on top of each other (blue dotted line). The averages of the waveforms are shown by the red lines. The figure demonstrates the fundamental similarity between the PPG signals recorded by the ADPD188GGZ and ADPD107 discrete solution.

Wavelet coherence functions were calculated to compare the two PPG signals. Based on the results analyzed in the case of all 11 subjects, no significant difference was observed in the frequency domain, nor in the phase domain between the two signals (see figure 2). 


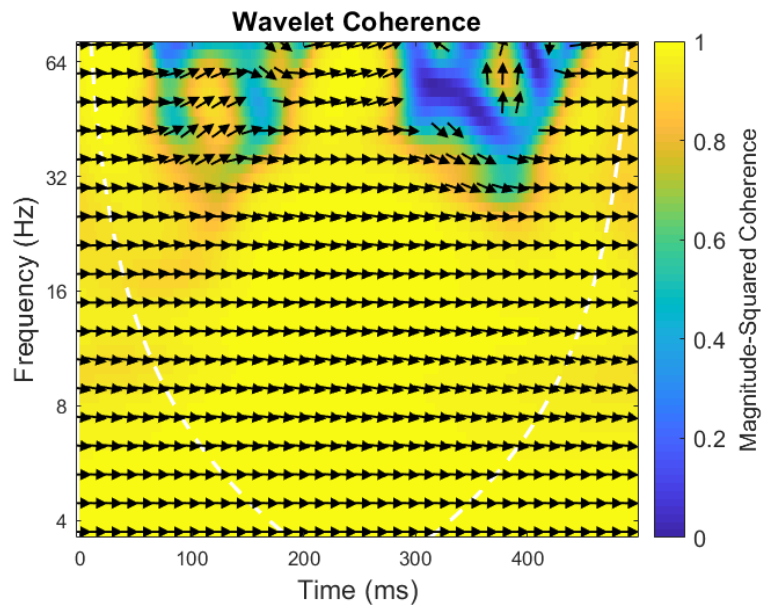

Figure 2. Representative visualization of the magnitudesquared coherence between the averages of the two compared PPG signals is shown by the color intensity plot in the time and frequency domains. Direction of the arrowheads is proportional with the phase difference between the signals. Horizontally pointing arrowhead to the right refers to no phase difference between the signals.

While developing a new product, it might also be helpful to look at the specific frequency bands, which can be extracted from a given signal, to be able to optimize the specifications.

Basic statistical properties of the magnitude-squared coherence between the two compared PPG systems were analyzed within all the relevant frequency ranges, as what is shown in figure 3 . The entire spectrum was divided into four specific frequency ranges between 0 and $20 \mathrm{~Hz}$ to analyze the variability of similarities between the signals.
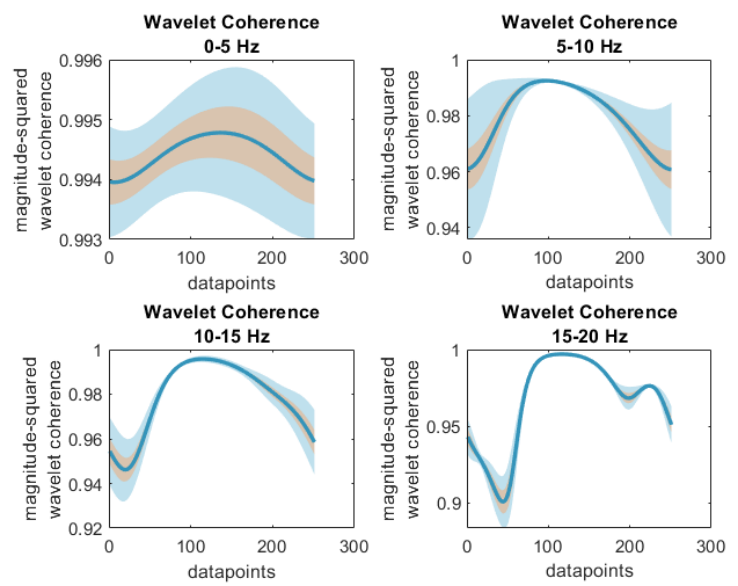

Figure 3.

Mean: blue line, variance: shaded by red, standard deviation: shaded by light blue, standard error of mean: shaded by yellow. Note that the mean is extremely high (strong coherence) and the standard deviation is extremely low especially around the peak of the PPG signal (the $125^{\text {th }}$ datapoint). The higher values of the magnitude-squared coherence refer to the more intensive similarity between signals. Data is shown only between $0-20 \mathrm{~Hz}$ for visualization purposes.

With this comparison, similarities between the two compared PPG systems were analyzed within all the relevant frequency ranges $(0-20 \mathrm{~Hz})$. For all the 11 subjects the coherence value was higher than 0.9 within all the explored frequency bands around the peak of the PPG signals, what tells us, that there is a very high similarity between the "gold standard" and the new integrated ADPD188GGZ.

Two different correlation-based methods were applied to continue the investigation at the deep data level. Correlation coefficients (R1) and the level of significance (P1), were computed between every individual PPG wave, following each other and the calculations were repeated also between every individual wave and the average (R2, P2).
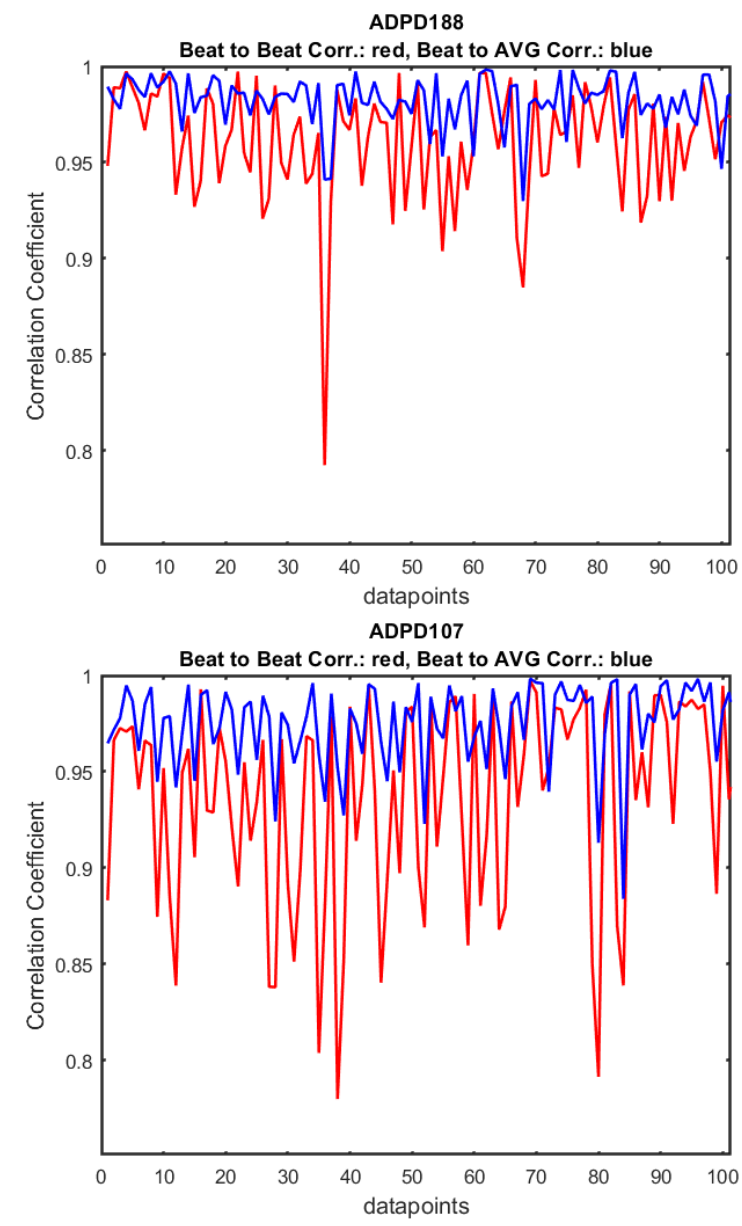
Figure 4. represents the time series of correlation coefficients (R1) and P values (P1), calculated between every individual PPG waveform, following each other (red). The correlation coefficients (R2) and P values (P2) were also calculated between every individual waveform and the averaged waveform (blue).

Based on the comprehensive correlation tests, we can conclude that it was not possible to observe any significant difference between the two systems, nor on the level of the individual waveforms (R1, P1), neither on the level of the individual waveforms versus averaged signals (R2, P2) (see Table 2.).

Table 2.

\begin{tabular}{|c|c|c|c|c|c|c|c|c|}
\hline & $\begin{array}{l}\mathrm{R} 1 \\
188\end{array}$ & $\begin{array}{l}\mathrm{R} 1 \\
107\end{array}$ & $\begin{array}{l}\mathrm{P} 1- \\
188\end{array}$ & $\begin{array}{l}\mathrm{P} 1 \\
107\end{array}$ & $\begin{array}{l}\text { R2 } \\
188\end{array}$ & $\begin{array}{l}\text { R2 } \\
107\end{array}$ & $\begin{array}{l}\mathrm{P} 2 \\
188\end{array}$ & $\begin{array}{l}\mathrm{P} 2 \\
107\end{array}$ \\
\hline \multirow{2}{*}{ ఫ్ } & 0.80 & 0.83 & 0.005 & 0.007 & 0.90 & 0.91 & 0.001 & 0.001 \\
\hline & 0.13 & 0.17 & 0.006 & 0.011 & 0.06 & 0.08 & 0.004 & 0.003 \\
\hline $\bar{v}$ & & & & & & & & \\
\hline
\end{tabular}

The table shows the mean and the standard deviation of the above described correlation coefficients (R1_188, R2_188 and R1_107, R2_107) and P values (P1_188, P2_188 and P1_107, P2_107) calculated for the PPG waveforms recorded by the ADPD188GGZ and ADPD107 sensor platforms.

\section{Conclusions}

Based on the results we can conclude, that the quality of the PPG signal recorded by the new ADPD188GGZ integrated optical module reaches the same performance level as the ADPD107 discrete module.

Moreover we can conclude, that the newly established Biosignal Metrology Standards (patent pending ID: P1900302) are appropriate to classify the quality of optical pulse volume signals, and that these standards establish the foundation for optimization of different usecases or fields of applications with specific technological solutions in order to support the usecase-specific health informatics application.

\section{References}

[1] Kais Gadhoumi et al., "A Statistical Comparative Study of Photoplethysmographic Signals in WristWorn and Fingertip Pulse-Oximetry Devices" CINC 2018

[2] Anna Shcherbina et al., "Accuracy in Wrist-Worn, SensorBased Measurements of Heart Rate and Energy Expenditure in a Diverse Cohort" J. Personalized Medicine, vol. 7, no. 3,
2017.

[3] Lien Desteghe et al., "Performance of Handheld Electrocardiogram Devices to Detect Atrial Fibrillation in a Cardiology and Geriatric Ward Setting" Europace, Clin. Res. Atraial Fibrillation, 19, 29-39., 2017.

Address for correspondence:

János Pálhalmi.

Ménesi u. 21. Budapest 1118 Hungary. EastWestData Ltd.

E-mail address: janos.palhalmi@eastwestdata.com 